\title{
Analysis of Chinese Consumer Behavior on Catering Services and Research on Corporate Marketing Strategies in the Post-Pandemic Era
}

\author{
Chen $\mathrm{Xu}^{1 *, \dagger}$, Zhilan Qian ${ }^{2, \dagger}$, Zihan Zhang ${ }^{3, \dagger}$ \\ ${ }^{1}$ Jiangxi University of Finance and Economics, Business 1 \\ ${ }^{2}$ Boston University, Business 2 \\ ${ }^{3}$ Rutgers University, Mathematics 3 \\ These authors contributed equally. \\ *Corresponding author. Email: zhilanq@bu.edu
}

\begin{abstract}
Since the COVID outbreak starting from February 2020, the Chinese catering industry has been facing an economic downturn. It is well-established by means of other research that the outdated business models that lots of catering businesses have of need to keep up with the contemporary dining consumption trend. Hence, this study aims to determine how the changes of consumer behaviors in the food sector after the pandemic can be better targeted in order to increase profits for the businesses. Specifically, it investigates the approaches to a better marketing strategy for businesses, which ultimately facilitates sustainable development of the Chinese catering industry in the long run. To analyze the trend in consumers' behavior, we took the business model of "Haidilao" as an example and integrate with the status quo of the Chinese. We come to the conclusion that people prefer to order food delivery online, prone to social media's promotions and various discounts. These results suggest that businesses need to carry out more customized product and promotion strategies that ensure food safety and quality, and at the same time, collaborating with internet influencers to attract more attentions and thus boost sales. In terms of pricing and placing strategy, it's recommended that businesses carry out a more efficient supply chain to cut down costs and also develop various channels to branch out their side businesses, creating a higher profit margin. Thus, the Chinese catering industry is expected to achieve an optimal development pattern and continues making a great contribution to the country's GDP.
\end{abstract}

Keywords: Post-pandemic Era, Catering industry, characteristics of consumer behavior, Marketing strategies

\section{INTRODUCTION}

China's catering market has been growing by leaps since the reform and opening-up strategies adopted since the late 1980s. According to the National Statistics Bureau of China, the scale of the catering industry was at a steady compound annual growth rate of $10.67 \%$ during the past ten years, and the annual output value took about $4.7 \%$ of the national GDP in the past five years. In 2019 alone, the catering industry's revenue reached 4.67 trillion RMB, increasing by $9.4 \%$ compared to the previous year, which was higher than the national GDP growth rate by approximately $3 \%$ [1].

However, the outbreak of Coronavirus at the end of 2019 exerted a direct and tremendous impact on the
Chinese catering industry, especially small and mediumsized enterprises. These enterprises have faced huge challenges such as a sharp decline in customer base and shortage of supply chain. Deloitte China partnered with the China Culinary Association has conducted research from mid-February to early March in 2020 and found out that $94 \%$ of the surveyed catering companies' services are negatively affected by the pandemic. Among them, 69\% of them have an over $80 \%$ reduction in dine-in customers. The main pressure on the cash flow that companies are facing derives from salary costs, cash withdrawn, and the rent. Over $80 \%$ of the surveyed companies expect additional financing in the upcoming 6 months [2]. This showcases the predicaments and burden that businesses have been undergoing, which foreshadows the urgent of cross-functional analysis on the transitions of consumer 
behaviors with regards to catering services and the problems businesses face.

Liang and Xing have conducted research on the impact of the pandemic on Chinese catering industries. They pointed out a variety of existed problems in the industry, including little progression in food security, the monotonous profit model, and lack of human resources. Thus, they bring up measures such as prioritizing food security via stricter supervision, developing omnichannel and a more well-rounded supply chain, and establishing a timely crisis management system [3]. Similarly, Xia Biqin and Wang Jiaxiang analyze the outdated business model that most of the businesses have been using, which turns out to be shaky after the tremendous decrease in the number of dine-in customers [4]. Likewise, Ding Zhichao utilizes statistics from Tianjin Shenzhou Shanglong Technology Co., Ltd to illustrate a sharp decline in sales revenue, which underlines the necessity for businesses to transform the traditional business model to better adapt to the large demand for food and grocery delivery [5]. All three studies articulate the root problems in detail that the catering industry is facing and briefs possible solutions that align with the government's policies to resume the predominant achievements that it originally did. However, all of them are lack statistical proof of consumers' behaviors. The studies take the perspective of business operators and governors in the field but fail to dive deeper into the customer's preferences and changes in their consumption behavior post-COVID. To better conduct a business model that suits the present market so that the business can adjust its marketing strategy accordingly, one must be insightful of the characteristics of consumers' behavior post-COVID and spot the changes.

Hence, this paper puts an emphasis on analyzing the behavioral statistics of in the catering industry during the post-pandemic era so that catering businesses can take advantage of the changes to make adjustments to their marketing strategy and internal operations. Studying from precedent cases of local businesses like "Haidilao" the behavioral statistics of leveraging niche online channels like "WeChat," "Taobao," and "Tik Tok," the Chinese catering industry will be expected to have a promising development and another boom in the near future.

\section{METHODOLOGY}

This paper mainly uses the method of literature research, case analysis, and summarization, and induction.

Firstly, literature research is the method of collection, identification, collation of literature, and the study of literature to form a scientific understanding of the facts. We refer to several literatures from CNKI (China National Knowledge Infrastructure), finding that most of that expound on the effect of COVID-19 on Chinese catering industry and its supply chains from different angle based on this, putting forward some ideas to improve that. However, the above literature virtually mentioned the impact of the epidemic on consumers consumption behavior, and thus inspired the strategy formulation of the thorough the consumers' consumption behavior. So we want to focus on it to fill the gap. Besides, we also take a reference to some industrial reports. The figures, like GDP, market size, quarterly revenue, passenger flow, and rate of return to work, are all from the National Bureau of Statistics, China Cuisine Association, and China Chain-Store \& Franchise Association. Through that, we basically know the current situation of catering industry and the problems they are facing with. Of course, theories about marketing are also involved with the help of Philip Kotler's Principles of Marketing.

Secondly, we use case analysis, it is a scientific analysis method that makes a thorough and careful study of representative things (phenomena)so as to obtain an overall understanding. And Haidilao hot pot, a famous catering enterprise in China, is our main research object. We want to use the "4P Theory" [6] to analyses the marketing strategies of Haidilao in the Post-COVID-19 Era and then seek out the problems of the strategies. However, there are some problems, such as the ambiguous market positioning of semi-finished products, the lack of market data, and the difficulty matching ecommerce and logistics platforms. And the low acceptance of popularization, publicity. Moreover, when expanding offline stores, developing small brands should pay attention to the local food culture. As well as the creativity, positioning, cost of the Internet publicity should also be considered

Finally, we make use of summarization and induction, which is an analytical reasoning method to discover general rules through observation of many special cases. We look through some literature and reports in regard to the consumption characteristics of consumers in the PostCOVID-19 Era. Then, we compare it with the strategies and problems of Haidilao we mentioned. After that, we can put forward some suggestions for catering industry based on changed consumption characteristics.

\section{RESULT}

\subsection{The status quo of the catering industry in the post-epidemic era}

Since China is one of the most populous countries globally, the total income of the catering industry is very optimistic before 2019. However, the new crown epidemic in early 2020 has greatly impacted China's catering industry. From the Spring Festival to the present, the operating income of catering merchants has dropped significantly; during the epidemic period, $78 \%$ of catering companies' turnover exceeded $100 \%$ at any time; $9 \%$ of 
enterprises had operating losses of more than $905 ; 7 \%$ of enterprises had operating losses of $80 \%$ However, only $5 \%$ of companies have a loss of less than $70 \%$ of their turnover.

As we all know, the Spring Festival should be the golden week for catering consumption. Due to the impact of the epidemic, most banquets have been unsubscribed. The Chinese Cuisine Association estimates that since the 2020 Spring Festival, the cancellation rate for New Year's Eve dinner alone has been as high as $94 \%$. Simultaneously, because it is the peak consumption season around the Spring Festival, catering companies will prepare more than three times the usual stocking volume of raw materials. Due to the suspension of business and the sharp decrease in passenger flow, a large amount of stored food been lost. Meanwhile, for most catering companies, the financial support they receive is far from enough to support their development. Companies with small operating scales and insufficient cash flow will obtain funds through indirect financing methods such as bank loans. Still, but its limitation is that banks have higher loan thresholds for small. Therefore, many small, medium, and micro enterprises cannot successfully raise funds from banks and other financial institutions.

On the contrary, although on the surface it seems that the epidemic has brought many indelible negative effects on China's catering industry, it has also helped accelerate the elimination of a large number of inferior catering companies while destroying it, and at the same time, it has brought more to the catering industry. Comprehensive development and innovation opportunities. Meanwhile, the impact of the epidemic also made many people aware of food safety issues. People began to pay more attention to food hygiene, not eating or touching ingredients that do not have safety standards. Furthermore, due to the epidemic, people have gradually become more inclined to choose takeaways and online shopping. Instead of eating directly in the store. Therefore, companies and merchants can also make corresponding improvements in their marketing strategies through these subtle changes of the public. It is obvious that the support and appeal of the government alone cannot help catering companies Really return to normal profitability. Through this epidemic, companies and businesses can also find problems in their industries and make corresponding improvements.

\subsection{Behavioral characteristics of catering consumers in the post-epidemic era}

Constrained by the restrictions of the home, consumers' catering consumption has shifted from dinein to home-based food, and in response to this, new demands and consumption habits have arisen accordingly. First of all, online consumption and logistics distribution have become the most fundamental consumer demand for the catering industry. Secondly, food and beverage categories need to be diversified. From the initial fast-food takeaway, fresh food, fruits, to later various online celebrity foods, finished dishes, etc., almost all food and beverage categories are covered. At the same time, the economic pressure brought about by the epidemic affects all walks of life, so more and more people are beginning to consciously save money and take money in their hands to plan ahead. Third, the increased demand for food safety requires not only food safety, but also delivery safety, so non-contact delivery has emerged, and so on.

\subsubsection{In the post-epidemic era, people are more inclined to shop online and take out}

First, online buying habits have expanded. After more than a month of living at home, many silver-haired elderly people joined the ranks of online catering and realized the convenience of online shopping. The formation of this habit has expanded the number of online consumers, and at the same time, it will also reduce the sales of offline stores. Stimulated by this epidemic, the online consumption scene will be further strengthened in the future. Food delivery will account for an increasing proportion of brand revenue. It is necessary for catering specialized operations for food delivery. At the same time, in order to respond to the call of the country, most citizens choose to order takeaway or online video shopping to reduce personnel contact. Moreover, under the impact of the epidemic, with the sharp decline in the demand for dining out, the consumer behavior and consumption structure have changed, and a large number of young people have joined the ranks of home cooking. The demand for simple retail catering is increasing rapidly.

\subsubsection{The information age promotes the development of the catering industry}

Moreover, due to the closure of the city, most people stay at home and cannot go out, so more and more people have downloaded social apps such as Tik Tok and Taobao. And these apps usually have celebrities, and Internet celebrities live streaming their goods, which also caused a wave of trends. More and more people choose to watch and buy products on these social apps. Nowadays, information is fragmented, short videos have become a major outlet, and Tik Tok is one of the best. In short video software, the trend has become a way for people to catch up with the trend. For example, in the catering industry, many users recommend Haidilao's new eating methods through Tik Tok, such as "self-made tomato beef noodles" and "mushroom stuffed meat". Such "net celebrity delicacies" are stimulating consumers' desire to experience. Second, due to the impact of the epidemic, consumers are paying more attention to food safety. And these social apps, such as Tik Tok, provide a platform for many businesses to record the purchasing process and food 
processing process through short videos and show them to consumers. This makes it open and transparent, allowing customers to be more assured and more proactive in consumption.

\subsubsection{Promotions and preferential activities can better meet customer needs}

In response to the country's call to fight the epidemic, most people lived apart from home during the epidemic. To avoid large-scale population movements and gatherings. This also means that not only the profitability of the has been threatened, but the income of all walks of life around the world has been hit hard. Because of this epidemic, more people have realized the importance of saving money. People in the post-epidemic era are more willing to spend money where they should be spent, rather than spending unscrupulously. Therefore, people are paying more and more attention to the benefits that discount promotions and preferential activities can bring to them. In other words, more people are willing to spend money on promotional activities and preferential activities. Therefore, according to consumers' different levels of consumption, different grades of cost-effective in-store purchasable products are presented to stimulate consumers' return, and at the same time, increase the rate of consumer return. Then use social platforms such as Moments and Weibo to share likes and participate in instore gifting activities to attract more potential users to participate.

\subsection{Problems in the marketing strategy of the catering industry-take Haidilao as an example}

Take Haidilao, a well-known catering company in China, as an example. In response to the country's calling to fight the epidemic, most people stayed at home during the epidemic to avoid large-scale population movements and gatherings, and the number of dine-in meals was greatly reduced. According to the company's financial report for 2019, dine-in business is Haidilao's main source of business. Haidilao's restaurant operating income in 2019 was 25.588 billion yuan, accounting for $96.3 \%$ of its revenue. Under the influence of the epidemic, the flow of dine-in customers has dropped significantly. After the epidemic has eased in some cities, the recovery of dine-in food in stores has been relatively slow. During 2020 epidemic, in order to stand firm during epidemic and prevent further losses, Haidilao chose to further accelerate its some other catering companies to ensure its own profitability. According to statistics, in the first half of 2020, Haidilao still opened 173 stores during the downturn of catering. On average, a new store opens every 1.05 days [7]. At the same time, comparing the number of net store openings in 2018 and 2019, the speed of store openings in 2020 will be faster than in the past two years. In addition to accelerating its store opening, Haidilao has also promoted the layout of many new brands at the same time continuously expanding its coverage in the entire catering industry through selfcreation, acquisitions, and other methods. At the same time, Haidilao also launched a takeaway business after the epidemic eased. Nevertheless, due to the inherent thinking of consumers that the most ideal way to eat hot pot is dine-in; second, the appearance of fresh meat after packaging is severely damaged, which will affect the image of the brand; third, the cost of hot pot takeaway is high and the packaging cost, Tableware costs, pot cleaning costs, etc., exacerbated its costs. Indeed, although the takeaway business can help Haidilao make some small profits during the epidemic, this may increase its loss in the long run. Furthermore, in order to make up for the losses during the epidemic, Haidilao has now tapped a new market for eating at home, that is, semifinished vegetables. By contrast, during the epidemic, Haidilao's decline in the turnover rate, the decline in single-store revenue, and the decrease in net profit margins are all issues that cannot be ignored.

\section{DISCUSSION}

\subsection{Product strategy}

During the epidemic period, semi-finished products became "hot cakes", which accelerated the development of the semi-finished product sped up Haidilao's original plan to launch formal semi-finished products [8]. However, although the development of the semi-finished products market is accelerating, science popularization, publicity, and the acceptance of are still not well. Hence, enterprises need to make efforts in creating characteristic products, positioning accurate service objects, and other aspects. And because of the epidemic, consumer behavior has changed a lot, and they're relying more on the Internet to get more information about products, and they are more trusting in the goods promoted by favorite stars, so enterprises can combine product end with channel end With the help of short video and e-commerce platform, such as Tik Tok, Taobao, and Amazon, enterprises can sell their products better.

Besides, consumers have become more concerned about food safety because of the epidemic. The older generation may be tempted to compare them with fast consumer goods like instant noodles, and when it comes to semi-finished products, the enterprises need to pay more attention to the propaganda of nutrition and health. We believe that the first to make small breakthroughs in this area will quickly occupy the market. Of course, brand, user stickiness, and other aspects enterprises also need to carefully care about.

\subsection{Price strategy}

As the epidemic has swept the world and affected all walks of life, people's awareness of crisis prevention has been significantly improved. People are more inclined to 
save money and hold it in their hands so that they can respond more calmly in the face of crisis. Haidilao has previously adopted a discount pricing strategy. They provide 31 percent discount for college students at special moment and discount vouchers and coupons for social groups, such as $¥ 30$ against $¥ 100$ vouchers, etc [9]. Now this strategy can obviously be continued, but the time period can be reduced for cost reasons, at the beginning of the epidemic, Haidilao raised the price of all its dishes, but it was condemned by society, and finally Haidilao apologized and adjusted the price back to the original position. In fact, we can pay more attention to the adjustment of supply chain and procurement and control the price at the cost end.

\subsection{Promotion strategy}

The epidemic has once again accelerated network live broadcasting, web celebrity economy, and a short video. Consumers gradually shift their consumption center to the Internet. Therefore, enterprises need to have their own space on the Internet to interact and communicate with consumers. Haidilao WeChat push follows the 4I principle: interest, interest, personality, and interaction [10]. But at the same time, they should also pay attention to integrity and quality. Consumers will consider these details in today's competitive situation of homogeneous products.

\subsection{Place strategy}

When Haidilao is expanding and creating a new brand, what needs to be paid attention to is whether it can meet the food culture of local people or people in that region. The epidemic has made people pickier about their consumption, and enterprises need to pay more attention to differentiated development strategies. Therefore, regardless of cash flow and cost, other enterprises should also pay attention to how likely they are to enter and explore the market when expanding their channels. Moreover, the takeout side also needs to be given enough attention, young consumers are particularly reliant on takeout. Therefore, if conditions permit enterprises can develop their own takeout teams just like KFC so as to give consumers the best experience and ensure the temperature, appearance and quality of food.

\subsection{Consumer behavior strategy}

Due to the development of the food delivery industry in China in recent years, more consumers are inclined to choose food delivery rather than in-store dining. Because takeaway allows people to choose their favorite food without leaving home, and for some popular restaurants, takeaway can also help people avoid spending a long time waiting in line. Hence, in order to cater to consumers' current consumption behavior, some large-scale catering companies such as Haidilao have begun to carry out online ordering and door-to-door services. Although hot pot takeaway has many inconveniences, it can increase the number of hot pot takeaway orders by stimulating consumers' desire.

On the contrary, Haidilao's sales increase due to the inconvenience of suspending the takeaway business and hot pot takeaway during the closure of the business, but at a slower rate than other restaurants. The inconvenience is as follows: the cost of hot pot takeaway business High, packaging fees, tableware fees, pot cleaning fees, etc. increase costs; also, eating is inconvenient, takeaway itself has the advantage of convenience, but hot pot takeaway does not reflect its convenience, it needs to recycle pots and induction cookers, and the delivery is more cumbersome, which is contrary to the original intention of takeaway.

\section{CONCLUSION}

The epidemic had a great impact on characteristics of consumer and prompted catering enterprises to improve their marketing strategies. Firstly, people pay more attention to food safety and environmental safety. Secondly, both the old and the young begin to take-out food gradually, the consumption structure. Secondly, consumers prefer to watch network broadcast and buy food through their favorite stars or anchors. Of course, the food here has changed too because of the epidemic. More and more semi-finished product has emerged, and their convenience has quickly made them a hot seller during the epidemic. Certainly, because of the impact of the epidemic on the world economy, people are beginning to have a crisis feeling about their money, and they are also becoming more cautious about spending, hoping to save enough money to face the uncertain situation.

Therefore, we can see that catering enterprises have some problems on the marketing strategies. For example, the nutritional health problems of semi-finished products; Quality of takeaway services; The product promotion through internet celebrity economy does not achieve precise positioning and differentiation; The lack of adequate measures to promote consumption; As well as the lack of sufficient interaction with consumers to increase consumer stickiness.

In terms of strategy, enterprises need to continuously invest in research and develop technology, to improve the rationality and market share of semi-finished products, and if the strength allows, enterprises can develop their own takeout team to improve customers' purchasing experience. Secondly, enterprises need to take good care of the platform which is used for interaction with consumers. Such as the creation of interesting WeChat official accounts and fully explore their own customer positioning in order to design and develop accounts based on their preference. Finally, businesses need to take price promotions according to their own circumstances, such as 
offering discounts to specific groups of people for specific periods of time or offering discounts for VIP membership. Generally speaking, the catering industry has gone through the reshuffle of the epidemic, and enterprises need to make more efforts to cater to consumers, getting rid of the old, so as promote the new to better entrench themselves in the whole industry.

\section{REFERENCES}

[1] P. Liang, L.X. Xing, Research on the Impact of COVID-19 on the Catering Industry and Countermeasures, Economic \& Trade Update. (2020)

[2] Deloitte, The Impact of COVID-19 on China's Catering Industry's Finance and Operations. (2020)

[3] P. Liang, L. Xing, Research on the Impact of COVID19 on the Catering Industry and Countermeasures, Economic \& Trade Update. (2020)

[4] B. Xia, J. Wang, On the Countermeasures for the Development of China's Catering Industry in the Post-COVID-19 Era. Journal of Wuhu Institute of Technology. (2020)

[5] Z. Ding, The Dilemma and Breakthroughs of the Catering Industry Under the Impact of COVID-19. China Market. (2020)

[6] P. Kotler, G. Armstrong, S.H, Ang, Siew Meng Leong, Chin Tiong Tan, David K. Tse, Principles of Marketing. (2005)

[7] B.R. Yang, While Expanding While Making Huge Losses, Haidilao Continues to Advance its MultiBrand Layout. (2020)

[8] Z.X. Li, Haidilao Layout Again Semi-Finished Products in the Future Need to Work Hard in Four Aspects. (2020)

[9] W.B. Lin, Y.L. Liu, X.X. Wu, A Brief Analysis of Haidilao's Service Marketing Strategy -- Based on the Analysis of the Consumer Line. (2020)

[10] P.B. Chen, J. Wang, X.X. Tang, Research on the Catering WeChat Marketing Mode Under the Background of SoLoMo -- Taking "Haidilao" WeChat Marketing as an Example. (2020) 